\title{
A dual defensive role of CIITA against retroviral infections Giovanna Tosi ${ }^{1}$, Luisa Bozzo ${ }^{1}$, Elisabetta Pilotti ${ }^{2}$, Claudio Casoli ${ }^{2}$ and Roberto Accolla*1
}

\author{
Address: ${ }^{1}$ Department of Clinical and Biological Sciences, University of Insubria, Varese, Italy and ${ }^{2}$ Department of Clinical Medicine, Nephrology, \\ and Prevention, University of Parma, Parma, Italy \\ * Corresponding author
}

from 2006 International Meeting of The Institute of Human Virology

Baltimore, USA. 17-2I November, 2006

Published: 2I December 2006

Retrovirology 2006, 3(SuppI I):SI02 doi:I0.1 I86/I742-4690-3-SI-SI02

(C) 2006 Tosi et al; licensee BioMed Central Ltd.

We describe how CIITA exerts a dual role against retroviral infection. The first, classical role is the upregulation of MHC class II expression and thus the capacity to present viral antigens to $\mathrm{CD} 4+\mathrm{T}$ cells. The other, evolutionary new and fundamental role is to inhibit viral replication by blocking specifically the function of the viral transactivators. HIV-1 Tat is inhibited through the competition for cyclin T1 of the P-TEFb complex, whereas HTLV-2 Tax-2 is inhibited through a concerted action which may increase the binding affinity of the CIITA-NFY complex for Tax-2, displacing it from the viral LTR promoter. As expected, two distint sequences in the N-term region of CIITA mediate the inhibitory action on Tat and Tax-2, respectively. Of note, Tax-1 from HTLV-1 seems also to be inhibited by the same sequence that inhibits HTLV-2 Tax-2. Interestingly, only those CIITA fragments containing the minimal inhibitory domains that localize into the nucleus could exert an effective suppressive action. Taken together, our results indicate that CIITA is an extant molecular tool endowed with distinct evolving functions against retroviruses. These distinct properties of CIITA will shed new light on the molecular mechanisms of adaptive coevolution of hosts and pathogens and may be exploited to envisage novel therapeutic strategies aimed at counteracting retroviral infections. 